\title{
Antiproton-nucleus potentials from global fits to antiprotonic X-rays and radiochemical data
}

\author{
E. Friedman ${ }^{a}$, A. Gal ${ }^{a}$, J. Mareš ${ }^{b}$ \\ ${ }^{a}$ Racah Institute of Physics, The Hebrew University, Jerusalem 91904, Israel \\ ${ }^{b}$ Nuclear Physics Institute, 25068 Řěz, Czech Republic
}

\begin{abstract}
We report on global fits of optical-model parameters to 90 data points for $\bar{p}$ X-rays and 17 data points of radiochemical data put together. By doing separate fits to the two kinds of data it is possible to determine phenomenologically the radial region where the absorption of antiprotons takes place and to obtain neutron densities which represent the average behaviour over the periodic table. A finite-range attractive and absorptive $\bar{p}$-nuclear isoscalar potential fits the data well. Self-consistent dynamical calculations within the RMF model demonstrate that the polarization of the nucleus by the atomic antiproton is negligible.
\end{abstract}

PACS 13.75.Cs 14.20.Dh 21.10.Gv 36.10.-k

Keywords: $\bar{p}$-nuclear interaction, $\bar{p}$ X-rays, $\bar{p}$ single-nucleon absorption, RMF calculations

Corresponding author: E. Friedman, elifried@vms.huji.ac.il

tel: +972 2658 4667, fax: +97226586347

November 18, 2018 


\section{INTRODUCTION}

Experiments on strong-interaction effects in antiprotonic atoms usually involve measurements of X-rays from $\bar{p}$-atomic transitions, providing values of level shifts and widths, the latter either measured directly or indirectly via the observed yields. Global analyses of large data sets, in terms of an optical model approach, have provided parameters for $\bar{p}$-nucleus optical potentials $[1,2]$ which fit well all the data then available, close to 50 data points, across the periodic table. It was found that the radial region which is 'sampled' by the $\bar{p}$-atom data is approximately between the half-density nuclear radius and some $4 \mathrm{fm}$ outside of it. The data could be well fit by a strongly attractive and strongly absorptive isoscalar $\bar{p}$-nuclear potential $V_{\text {opt }}$, underlined by a $\bar{N} N s$-wave interaction, in which the imaginary (absorptive) part outweighs the real (attractive) part. Gradient ( $p$-wave) terms in $V_{\text {opt }}$ were not required by the $\bar{p}$-atomic data. Furthermore it was concluded, for any global fit of $\bar{p}$-atom data, that the isovector components of $V_{\text {opt }}$ were relatively insignificant, provided that single-particle densities which describe correctly the nuclear surface region and beyond were used. We note that since $V_{\text {opt }}$ is strongly absorptive, its extrapolation into nuclear-matter densities is highly model dependent. Even if a unique extrapolation were possible, the existence of well-defined nuclear bound states of antiprotons would have been unlikely due to the large widths expected.

The experimental situation has changed significantly with the availability of high-quality data for several sequences of isotopes along the periodic table due to the PS209 collaboration [3] which made it possible to perform global fits to larger and more accurate data bases consisting of close to 100 data points. A good description of these new data was achieved in global analyses by using a finite-range $V_{\text {opt }}$ in which a $\bar{p} N s$-wave interaction is folded with the nuclear density $\rho=\rho_{n}+\rho_{p}$, as reported preliminarily in Ref. [4]. Another type of experimental information on the $\bar{p}$-nucleus interaction in $\bar{p}$ atoms is obtained using the socalled radiochemical method. By studying the production of nuclei differing from the target nucleus by the removal of one neutron or one proton it is possible to obtain information on the ratios of $\bar{p}$ absorption on neutrons to $\bar{p}$ absorption on protons in the periphery of the target nucleus, at a distance about $2.5 \mathrm{fm}$ beyond the half-density charge radius according to Ref. [5]. At such distances the nuclear density is dominated by the neutron density which is not directly measured or model-independently determined, and it is vital to use reliable nuclear models rooted in a sound nuclear phenomenology to extract these densities for use in optical-model calculations. Close to 20 target nuclei have been studied by the radiochemical method [6,7] and in several cases [8-10] good consistency was found between the radiochemical data and the X-ray data, within an optical-model approach.

The present paper reports on global fits of optical potentials to all those experimental results, consisting of 90 points of X-ray data and 17 points of radiochemical data put together. Our aim in this paper is to determine the $\bar{p}$-nuclear interaction potential at the nuclear surface and beyond, while identifying simultaneously the largely unknown neutron densities which provide a good fit to the combined data in terms of $V_{\text {opt }}$ that depends sensitively on these densities. The organization of the paper is as follows. In section II we verify that the conventional approach of static atomic calculations, i.e. using static nuclear densities, is valid. This is achieved by performing dynamical calculations within a relativistic mean field (RMF) approach where the nucleus is allowed to be polarized by the atomic $\bar{p}$. Note 
that for $\bar{p}$-nuclear configurations, in contrast, dynamical RMF calculations for antiprotons [11] have yielded considerable nuclear polarization effects. Section III presents the $\bar{p}$-nucleus potential and the methodology of the present work, in particular as regarding the use of neutron densities. In section IV we present results, first only for the X-ray data, followed by results for the radiochemical data where we examine under what conditions the two sets of results are consistent with each other. Global fits to the combined data base of X-ray and radiochemical data are then presented and conclusions are drawn on the admissible neutron-density shapes. Section V summarizes the findings of this work.

\section{DYNAMICAL CALCULATIONS OF ANTIPROTONIC ATOMS}

Self-consistent RMF calculations of deeply bound $\bar{p}-$ nuclear states [11], as well as $\bar{K}$ nuclear states [12], revealed extremely large dynamical polarization effects. The core nucleus is highly compressed and its binding energy significantly increases due to the strongly attractive $\bar{p}$ - nucleus interaction. It is therefore mandatory to check to what extent polarization effects are important in the calculations of $\bar{p}$-atomic states and whether the static approach hitherto used in atomic calculations is justified. In order to address this problem, we performed self-consistent dynamical calculations of $\bar{p}$ - atomic states within the RMF model.

In the RMF framework the core nucleons and the $\bar{p}$ interact through the exchange of isoscalar-scalar $(\sigma)$ and -vector $(\omega)$, isovector-vector $(\rho)$, and Coulomb $(A)$ fields, which are treated in the mean-field approximation. The advantage of the RMF approach is that antinucleons $(\bar{N})$ are naturally included in the Lagrangian density and, consequently, the derivation of the relevant equations of motion is straightforward. The Dirac equation for nucleons and antinucleons $(i=N, \bar{N})$ reads:

$$
\left[-\mathrm{i} \vec{\alpha} \cdot \vec{\nabla}+\beta\left(M_{i}+S_{i}\right)+V_{i}\right] \Psi_{i}^{a}=\epsilon_{i}^{a} \Psi_{i}^{a},
$$

where $S_{i}=g_{\sigma i} \sigma, V_{i}=g_{\omega i} \omega^{0}+g_{\rho i} \tau_{3} \rho_{3}^{0}+e_{i} \frac{\left(1+\tau_{3}\right)}{2} A^{0}$, and $a$ denotes quantum numbers of single-particle states.

The presence of the $\bar{p}$ in the nuclear system under consideration modifies the source terms in the equations of motion for the meson fields:

$$
\begin{aligned}
\left(-\Delta+m_{\sigma}^{2}+c_{1} \sigma+c_{2} \sigma^{2}\right) \sigma & =-g_{\sigma N} \rho_{S N}-g_{\sigma \bar{N}} \rho_{S \bar{N}} \\
\left(-\Delta+m_{\omega}^{2}+d \omega_{0}^{2}\right) \omega_{0} & =g_{\omega N} \rho_{B N}+g_{\omega \bar{N}} \rho_{B \bar{N}} \\
\left(-\Delta+m_{\rho}^{2}\right) \rho_{3}^{0} & =g_{\rho N} \rho_{3 N}+g_{\rho \bar{N}} \rho_{3 \bar{N}} \\
(-\Delta) A_{0} & =e_{p} \rho_{p}+e_{\bar{p}} \rho_{\bar{p}}
\end{aligned}
$$

where $\rho_{S i}, \rho_{B i}, \rho_{3 i}, \rho_{j}(j=p, \bar{p})$ are the scalar, vector (baryon), isovector, and proton and antiproton densities, respectively.

While for nucleons the Dirac equation (1) was solved, for the antiproton we actually solved a Schrödinger equation with a complex $\bar{p}$ optical potential. The real part of the $\bar{p}$ potential, $\operatorname{Re} V_{\text {opt }}$, was constructed as the Schrödinger equivalent potential from the scalar and vector meson mean fields:

$$
\operatorname{Re} V_{\text {opt }}=S_{\bar{N}}+V_{\bar{N}}+\left(S_{\bar{N}}^{2}-V_{\bar{N}}^{2}\right) / 2 M .
$$


Note that, for antiprotons, both $S_{\bar{N}}$ and $V_{\bar{N}}$ are attractive owing to G-parity. When solving Eqs.(2) for the meson mean fields, we make the approximation $\rho_{S \bar{N}}=\rho_{B \bar{N}}=\rho_{\bar{N}}$, where $\rho_{\bar{N}}$ is the $\bar{p}$ density formed by solving the Schrödinger equation (3). This approximation amounts to neglecting a few percent difference at most between the scalar and vector densities, a neglect that turns out to be completely insignificant for the dynamical effects calculated in $\bar{p}$ atoms. The $\bar{p}$ optical potential is dominated by its imaginary term [2] which is due to the annihilation of antiprotons on nucleons. $\operatorname{Im} V_{\text {opt }}$ was taken proportional to the nuclear density $\rho(r)$ and its depth was adjusted to fit the atomic data. It is to be noted that in this dynamical approach the nuclear density $\rho$ is not a static quantity but is affected by the antiproton interacting strongly with the core nucleons via meson mean fields.

In our dynamical calculations of the $\bar{p}$-nucleus systems, the standard RMF Lagrangian with the nonlinear parameterizations TM1 [13] and due to Sharma et al. [14] were used. Applying the G-parity transformation as a starting point to determine the antinucleon $(\bar{p})$ couplings $g_{j \bar{N}}(j=\sigma, \omega, \rho)$ but realizing that the presence of strong annihilation channels and various many-body effects could cause significant deviations from the G-parity values, we introduced following Ref. [11] a reduction parameter $\varepsilon(\varepsilon \leq 1)$ to allow for departures from the ideal G-parity limit:

$$
g_{\sigma \bar{N}}=\varepsilon g_{\sigma N}, \quad g_{\omega \bar{N}}=-\varepsilon g_{\omega N}, \quad g_{\rho N}=\varepsilon g_{\rho N} .
$$

The reduction parameter $\varepsilon$ was determined by fitting the calculated energy shift and width of a particular $\bar{p}$ atomic state to the corresponding experimental result. Static calculations showed that fits to the atomic $\bar{p}$ data for ${ }^{16} \mathrm{O},{ }^{40} \mathrm{Ca}$ and ${ }^{208} \mathrm{~Pb}$ produced very nearly the same potential parameters as obtained from fits to the full data base (but with larger errors with which the optical-potential parameters are determined). Consequently we chose these three nuclei for checking polarization effects by studying the $3 d$ atomic state of ${ }^{16} \mathrm{O}$, the $4 f$ state of ${ }^{40} \mathrm{Ca}$ and the $9 k$ state of ${ }^{208} \mathrm{~Pb}$. The value of the reduction parameter $\varepsilon$ needed to fit the atomic data was between $0.15\left({ }^{40} \mathrm{Ca}\right)$ and $0.35\left({ }^{208} \mathrm{~Pb}\right)$. This indicates a large deviation of the $\bar{p}$ couplings from the G-parity values.

The coupled system of equations (1) for nucleons, (2) for the meson- and electromagnetic fields, and the Schrödinger equation for $\bar{p}$ was solved self-consistently by iterations. In order to check the numerical procedure, $\bar{p}$-nuclear states were calculated using the TM1 parameterization and the results of Ref. [11] were reproduced. Large polarization effects were found for $\bar{p}$-nuclear states, as expected. Subsequently, dynamical effects for $\bar{p}$-atomic states were studied. Comparing the energy shifts and widths calculated self-consistently with the predictions of the corresponding static calculations, we found totally negligible effects of less than $1 \mathrm{eV}$ for the above three $\bar{p}$-atomic states.

Finally, deeply bound $\bar{p}$-atomic states [15] were also calculated in ${ }^{16} \mathrm{O},{ }^{40} \mathrm{Ca}$, and ${ }^{208} \mathrm{~Pb}$. For illustration, Table I presents calculated binding energies $\left(B_{\bar{p}}\right)$ and widths $\left(\Gamma_{\bar{p}}\right)$ for the $1 s$ and $2 p \bar{p}$-atomic states in Ca, comparing between the results of dynamical and of static calculations. The differences of few eV, caused by the polarization effects, are exceedingly small and experimentally unobservable. We conclude that static analyses of $\bar{p}$-atomic data which neglect the rearrangement of the core nucleons are adequate. 


\section{METHODOLOGY}

In order to preserve the connection to previous studies of hadronic atoms [2], the KleinGordon $(\mathrm{KG})$ equation is used in the form

$$
\left[\Delta-2 \mu\left(B+V_{\mathrm{opt}}+V_{c}\right)+\left(V_{c}+B\right)^{2}\right] \psi=0 \quad(\hbar=c=1) .
$$

Here, $V_{c}$ denotes the static Coulomb potential for the $\bar{p}$ due to the finite charge distribution of the nucleus, including the first-order $\alpha(Z \alpha)$ vacuum-polarization potential, $\mu$ is the $\bar{p}$ nucleus reduced mass and $B=B_{\bar{p}}+\mathrm{i} \Gamma_{\bar{p}} / 2$ is the complex binding energy. The interaction of antiprotons with the nucleus is described here in terms of an optical potential $V_{\text {opt }}$ which in the simplest ' $t \rho$ ' form is given by

$$
2 \mu V_{\mathrm{opt}}(r)=-4 \pi\left(1+\frac{\mu}{M} \frac{A-1}{A}\right)\left[b_{0}\left(\rho_{n}+\rho_{p}\right)+b_{1}\left(\rho_{n}-\rho_{p}\right)\right]
$$

where $\rho_{n}$ and $\rho_{p}$ are the neutron and proton density distributions normalized to the number of neutrons $N$ and number of protons $Z$, respectively, $A=N+Z$, and $M$ is the mass of the nucleon. In the zero-range ' $t \rho$ ' approach the parameters $b_{0}$ and $b_{1}$ are minus the $\bar{p}$ nucleon isoscalar and isovector scattering lengths, respectively, otherwise these parameters may be regarded as 'effective' and are obtained from fits to the data, either as given above or in a finite-range folding model [4]. The explicit inclusion of an isovector term in $V_{\text {opt }}$ is motivated by the fact that much data now exist [3] for chains of isotopes and because the radiochemical method provides information on ratios of neutron to proton densities. A least-squares search procedure was used to adjust the potential parameters so as to obtain a best fit to the experimental shift and width measurements.

A note on wave equations is in order here. The quadratic term $\left(V_{c}+B\right)^{2}$ in the $\mathrm{KG}$ equation (5) has little effect on the calculated strong-interaction shift and width, and omitting it gives rise to the nonrelativistic Schrödinger equation. One might ask why use the $\mathrm{KG}$ equation as extension into the relativistic domain instead of the Dirac equation for the $\bar{p}$ fermion. Indeed when interpreting experimental transition energies in order to extract the strong interaction effects it is essential to use the Dirac equation with finite size nuclear charge distribution and vacuum polarization terms [16]. However, strong interaction effects in $\bar{p}$ atoms are normally given as the proper averages over the fine structure components. The use of the KG equation rather than the Dirac equation is numerically justified when fine-structure effects are negligible or are treated in an average way, as for the X-ray transitions considered here. The leading $j$ dependence $\left(j=l \pm \frac{1}{2}\right)$ of the energy for solutions of the Dirac equation for a point-charge $1 / r$ potential goes as $\left(j+\frac{1}{2}\right)^{-1}$, and on averaging it over the projections of $j$ gives rise to $\left(l+\frac{1}{2}\right)^{-1}$ which is precisely the leading $l$ dependence of the energy for solutions of the KG equation. The higher-order contributions to the spin-orbit splitting are suppressed by $O(Z \alpha / n)^{2}$ which is of order $1 \%$ for the high- $n$ X-ray transitions encountered for antiprotons. We checked numerically for few typical cases that the spin-orbit averaged shifts and widths thus obtained differ by less than $1 \%$ from the $(2 j+1)$-average of the corresponding quantities obtained by solving the Dirac equation. This difference is considerably smaller than the experimental errors placed on the measured X-ray transition energies and widths. 
The nuclear densities are an essential ingredient of the optical potential. The density distribution of the protons is usually considered known as it is obtained from the nuclear charge distribution [17] by unfolding the finite size of the charge of the proton. The neutron distributions are, however, generally not known to sufficient accuracy. A host of different methods have been applied to the extraction of rms radii of neutron distributions in nuclei but the results are sometimes conflicting, see Refs. [18-23]. For many nuclei there is no direct experimental information whatsoever on neutron densities and one must then rely on models. To complicate things further we note that there is a long history of conflict between values of neutron rms radii derived from experiments using hadronic projectiles and neutron rms radii obtained from theoretical calculations. For that reason we have adopted a semi-phenomenological approach that covers a broad range of possible neutron density distributions.

Experience with pionic atoms showed [24] that the feature of neutron density distributions which is most relevant in determining strong interaction effects in pionic atoms is the radial extent, as represented for example by $r_{n}$, the neutron density rms radius. Other features such as the detailed shape of the distribution have only minor effect. For that reason we chose the rms radius as the prime parameter in the present study. Since $r_{p}$, the rms radius for the proton density distribution, is considered to be known, we focus attention on values of the difference $r_{n}-r_{p}$. In previous analyses of $\bar{p}$ radiochemical data $[8,23]$ a linear dependence of $r_{n}-r_{p}$ on $(N-Z) / A$ was employed, namely,

$$
r_{n}-r_{p}=\alpha \frac{N-Z}{A}+\gamma
$$

with $\alpha$ close to $1.0 \mathrm{fm}$ and $\gamma$ close to zero. The same expression with $\alpha$ close to $1.5 \mathrm{fm}$ was found [24] to represent well results of RMF calculations [25] for stable nuclei, but these values of $r_{n}-r_{p}$ are larger by about $0.05-0.10 \mathrm{fm}$ than the 'experimental' values in mediumweight and heavy nuclei used in recent relativistic Hartree-Bogoliubov (RHB) versions of mean-field calculations [26,27]. Expression (7) has been adopted in the present work and, for lack of better global information about neutron densities, the value of $\alpha$ was varied over a reasonable range in fitting to the data. This procedure is based on the expectation that for a large data set over the whole of the periodic table some local variations will cancel out and that an average behaviour may be established.

In order to allow for possible differences in the shape of the neutron distribution, the 'skin' and the 'halo' forms of Ref. [8] were used, as well as an average between the two. Adopting a two-parameter Fermi distribution both for the proton (unfolded from the charge distribution) and for the neutron density distributions

$$
\rho_{n, p}(r)=\frac{\rho_{0 n, 0 p}}{1+\exp \left(\left(r-R_{n, p}\right) / a_{n, p}\right)},
$$

then for each value of $r_{n}-r_{p}$ in the 'skin' form the same diffuseness parameter for the protons and the neutrons $a_{n}=a_{p}$ was used and the $R_{n}$ parameter was determined from the rms radius $r_{n}$. In the 'halo' form the same radius parameter $R_{n}=R_{p}$ was assumed and $a_{n}^{\mathrm{h}}$ was determined from $r_{n}$. In the 'average' option the diffuseness parameter was set to be the average of the above two diffuseness parameters $a_{n}^{\text {ave }}=\left(a_{p}+a_{n}^{\mathrm{h}}\right) / 2$ and the radius parameter $R_{n}$ was then determined from the rms radius $r_{n}$. In this way we have used three shapes of 
the neutron distribution for each value of its rms radius all along the periodic table. Figure 1 shows as an example the densities for ${ }^{120} \mathrm{Sn}$ after a finite range folding (see below). The relevant radial region for the $\bar{p}$-nucleus interaction is $8-9 \mathrm{fm}$ in this case, and it is seen that the neutron densities are quite different for the three models.

Returning to the optical potential Eq.(6), we note that in Ref. [4] it was found that the $\mathrm{X}$-ray data could be described better with a finite-range interaction, where the densities of Eq.(6) were replaced by 'folded' densities

$$
\rho^{F}(r)=\int d \mathbf{r}^{\prime} \rho\left(\mathbf{r}^{\prime}\right) \frac{1}{\pi^{3 / 2} \beta^{3}} e^{-\left(\mathbf{r}-\mathbf{r}^{\prime}\right)^{2} / \beta^{2}}
$$

with the value of $\beta=0.85 \mathrm{fm}$ used for both the real and imaginary parts of the potential. For the analysis of the radiochemical data we adopt the approach of Refs. [6-8] that the method is sensitive to the neutron to proton density ratio close to $2.5 \mathrm{fm}$ outside of the halfdensity radius of the charge density [5]. The experimental ratios of absorption on neutrons to absorption on protons were therefore compared to

$$
\frac{\operatorname{Im}\left(b_{0}+b_{1}\right) I_{n}}{\operatorname{Im}\left(b_{0}-b_{1}\right) I_{p}}
$$

where $I_{n, p}$ are the volume integrals of the neutron and proton densities, respectively, either between 2.0 and $3.0 \mathrm{fm}$ or between 2.5 and $3.5 \mathrm{fm}$ outside of the half-density radius of the charge density. For a finite-range potential the folded densities were used. No use was made of atomic wavefunctions in calculating the ratios because their effect largely cancel out in the ratios. Choosing the range of integration was guided by the conclusions of Ref. [5] which obviously were based on atomic wavefunctions. By requiring consistency between radiochemical data and X-ray data we could independently check the conclusions of Ref. [5] as explained below.

\section{RESULTS AND DISCUSSION}

\section{A. X-ray data}

A brief report on global fits to the present extended X-ray data has already been published [4]. The data base included strong interaction level shifts and widths for antiprotonic atom levels in ${ }^{16,18} \mathrm{O},{ }^{40,42,43,44,48} \mathrm{Ca},{ }^{54,56,57,58} \mathrm{Fe},{ }^{58,60,62,64} \mathrm{Ni},{ }^{90,96} \mathrm{Zr},{ }^{106,116} \mathrm{Cd},{ }^{112,116,120,124} \mathrm{Sn}$, ${ }_{122,124,126,128,130} \mathrm{Te}$ and ${ }^{208} \mathrm{~Pb}$, a total of 90 points [3]. It was found that by introducing a finite range into the interaction within the folding-model approach of Eq.(9) consistently better fits to the data were obtained compared to the corresponding zero-range approach.

Figure 2 shows values of $\chi^{2}$ obtained for the three shapes of neutron distributions discussed above as function of the slope parameter $\alpha$ in the expression for $r_{n}-r_{p}$, Eq.(7), using a finite-range parameter of $\beta=0.85 \mathrm{fm}$. It is seen that both the 'halo' model and the 'average' model for $\rho_{n}$ produce very acceptable fits with $\chi^{2}$ per point of about 2.2 . Recall that values of $\alpha$ are likely to be between 0.9 and $1.3 \mathrm{fm}$, as the value of $1.5 \mathrm{fm}$ found from fits to results of RMF calculations is now regarded as an over-estimate. The results for the 'halo' version confirm earlier findings [8] that for this shape of neutron density agreement with 
experiment is obtained for $\alpha$ very close to $1 \mathrm{fm}$. For the 'skin' variety of $\rho_{n}$ the minimum of $\chi^{2}$ is obtained for a value of $\alpha$ close to $2 \mathrm{fm}$ which is unreasonably large [23,26,27]. The 'average' type of $\rho_{n}$ is quite acceptable both in the quality of fit and in the value of $\alpha$ at the minimum of $\chi^{2}$. For this model the slope of $\rho_{n}$ is smaller at large radii than the slope of $\rho_{p}$, as expected on grounds of binding energies, yet the model is not restricted in having the same half-density radii for the proton and for the neutron distributions. Note that from global fits to strong interaction shifts and widths in pionic atoms it is found [24] that the 'halo' shape is rejected, whereas the other two shapes are quite acceptable. However, the pionic atom data are sensitive to considerably smaller radii (and consequently larger densities) than is the case for $\bar{p}$ atoms. Figure 3 shows values of the isoscalar amplitude $b_{0}$ from fits to the data for the 'average' type of neutron density using a finite-range interaction. The isovector amplitude was found to be consistent with zero [4] and we return to this point below.

\section{B. Radiochemical data}

Experimental ratios of $\bar{p}$ absorption on neutrons to absorption on protons were taken from Refs. [6,7]. Initial calculations showed that very large contributions to the resulting $\chi^{2}$ came from ${ }^{106} \mathrm{Cd}$ and ${ }^{112} \mathrm{Sn}$ and subsequently these two nuclei were excluded from the data set. Possible explanations for the problem with these two nuclei in terms of a $\bar{p} p$ quasi bound state are given in Ref. [28]. We have therefore used 17 values of absorption ratios for the following nuclei: ${ }^{48} \mathrm{Ca},{ }^{58} \mathrm{Ni},{ }^{96} \mathrm{Zr},{ }^{100} \mathrm{Mo},{ }^{96,104} \mathrm{Ru},{ }^{116} \mathrm{Cd},{ }^{124} \mathrm{Sn},{ }^{128,130} \mathrm{Te},{ }^{144,154} \mathrm{Sm}$, ${ }^{148} \mathrm{Nd},{ }^{160} \mathrm{Gd},{ }^{176} \mathrm{Yb},{ }^{232} \mathrm{Th}$ and ${ }^{238} \mathrm{U}$. With the potential parameter $b_{1}$ consistent with zero the ratios Eq.(10) become independent of the parameters of the potential, but they are quite sensitive to values of $r_{n}-r_{p}$. This is seen clearly in Fig. 4 where $\chi^{2}$ values for the radiochemical data are shown as function of $\alpha$ for the 'halo' and for the 'average' types of neutron densities. With the proton density held fixed the calculated ratios obviously depend on the neutron density and on the radius where the ratios of densities are calculated. To avoid the use of densities at a point we have integrated the densities over a distance of $1 \mathrm{fm}$, and Fig. 4 shows results for integrations between 2.5 and $3.5 \mathrm{fm}$ and between 2 and $3 \mathrm{fm}$ beyond the half-density radius of the charge distribution. However, due to the exponential decrease of the densities at such large radii the integrals are dominated by the densities close to the lower limit of the range of integration.

Comparing positions of the minima in Fig. 4 for the radiochemical data with Fig. 2 for the X-ray data, we note a consistency between the two regarding the parameter $\alpha$ when the integration range for the radiochemical data is 2.5 to $3.5 \mathrm{fm}$ beyond the charge radius, whereas moving that range down by $0.5 \mathrm{fm}$ leads to inconsistency between the two types of data. This is a significant test because both types of data depend differently on the nuclear densities. This result confirms in a phenomenological way the theoretical conclusion of Wycech et al. [5] that most of the absorption takes place close to $2.5 \mathrm{fm}$ beyond the charge radius. We therefore adopt the 2.5 to $3.5 \mathrm{fm}$ segment as the integration range for the global analysis of the combined X-ray and the radiochemical data. 


\section{Global fits to X-ray and radiochemical data}

Fits to all the 107 data points due to the X-ray and radiochemical data were made, using the various shapes for the neutron densities as described above and varying the isoscalar potential parameter $b_{0}$ and the isovector parameter $b_{1}$. Finite-range folding was assumed throughout with a range parameter $\beta=0.85 \mathrm{fm}$. For the radiochemical data the integration range was chosen between 2.5 and $3.5 \mathrm{fm}$ beyond the charge half-density radius, as described above. The real part of $b_{1}$ was always found to be consistent with zero and subsequently it was excluded from the fits. Figure 5 shows results for the 'average' shape of the neutron densities and Fig. 6 shows similar results for the 'halo' shape. In the lower part of the figures we show values of $\chi^{2}$ for the X-ray data separately and for the combined X-ray and radiochemical data. The upper part shows the potential parameters.

It is seen that although $\operatorname{Im} b_{1}$ assumes non-vanishing values away from the minima of $\chi^{2}$, at the minima this parameter is essentially zero. Accepting those minima for the 'halo' and for the 'average' shapes with $\alpha$ between 0.9 and $1.3 \mathrm{fm}$ as a fair representation, on the average, of neutron densities, we end up with the values of $b_{0}$ as found in Ref. [4], namely $\operatorname{Re} b_{0}=1.3 \pm 0.1 \mathrm{fm}, \operatorname{Im} b_{0}=1.9 \pm 0.1 \mathrm{fm}$, with a folding range of $\beta=0.85 \mathrm{fm}$.

The vanishing of $\operatorname{Im} b_{1}$ at the best-fit points for the two acceptable models for neutron densities deserves a comment. We note that the four $\bar{N} N$ potentials considered in Table 3 of Ref. [1] yield for $\operatorname{Im} b_{1}$ values which are a factor of 10-20 smaller than the corresponding values of $\operatorname{Im} b_{0}$, so it is conceivable that also for the effective phenomenological parameters a similar situation will hold. With the many groups of isotopes in the present data base the dependence on $N-Z$ must play a role. The neutron densities are obviously properly normalized, but the rms radii are not set a priori and they are found, on the average, in the process of $\chi^{2}$ fits. The vanishing of $\operatorname{Im} b_{1}$ is presumably an indication of the general validity of the neutron densities used here. As a test we note that for the unacceptable 'skin' model, where $\alpha$ is close to $2 \mathrm{fm}$ at the minimum, a non-vanishing value is found for $\operatorname{Im} b_{1}$, which presumably compensates for the unphysical neutron densities.

The fits shown in Figs. 2 - 6 can be marginally improved, without changing the conclusions of the present work, if the parameterization of $r_{n}-r_{p}$ in Eq. (7) is slightly modified to incorporate the expected $A$ dependence of the parameter $\gamma$ due to the increased Coulomb repulsion within the $N=Z$ core. To this end we have used $\gamma=-0.0162 A^{1 / 3}$ fm to interpolate between $r_{n}-r_{p}$ values in ${ }^{16} \mathrm{O},{ }^{40} \mathrm{Ca}$ and the $\mathrm{Sn}$ and $\mathrm{Pb}$ isotopes within recent $\mathrm{RHB}$ calculations [26] which yield values for the $(N-Z) / A$ slope parameter $\alpha \sim 1.5-1.6 \mathrm{fm}$. This analysis, again, strongly suggests that the prefered neutron densities are best described by the 'average' type shape. A more expanded discussion of these results, plus related ones for pionic atoms, are relegated to a separate publication.

\section{CONCLUSIONS}

In conclusion, self-consistent dynamical calculations within the RMF model demonstrate that the polarization of the nucleus by the atomic antiproton is negligible, thus confirming the validity of the common static approach in which the nuclear polarization is disregarded. We have performed, for the first time, global fits of optical model parameters to 90 data points for antiprotonic X-rays and 17 data points of radiochemical data put together. With 
the help of separate fits to the two kinds of data we could verify in a phenomenological way the theoretical prediction [5] for the radial region where most of the absorption of atomic antiprotons takes place. Despite the use of many groups of isotopes all along the periodic table, the parameters of the isovector part of the potential could not be determined in global fits, and are therefore assumed to be consistent with zero. The optical potentials are determined at radii where the density is $2-3 \%$ of the central nuclear density. It is therefore rather meaningless to extrapolate these potentials to the nuclear interior. However, if extrapolated with the nuclear densities used in the present work, then at the center of the nucleus the potential is attractive with about $110 \mathrm{MeV}$ depth, and its absorptive part is close to $160 \mathrm{MeV}$ deep. While this attraction is fairly strong, about twice that for nucleons in nuclear-matter densities, it is much weaker than naive G-parity arguments suggest. The world's data on $\bar{p}$-nucleus interaction potential are well accounted for with the effective parameters $\operatorname{Re} b_{0}=1.3 \pm 0.1 \mathrm{fm}, \operatorname{Im} b_{0}=1.9 \pm 0.1 \mathrm{fm}$, with a folding range of $\beta=0.85 \mathrm{fm}$.

This work was supported in part by the Israel Science Foundation grant 131/01 and by the GA AVCR grant IAA1048305. 


\section{REFERENCES}

[1] C.J. Batty, E. Friedman, A. Gal, Nucl. Phys. A 592 (1995) 487.

[2] C.J. Batty, E. Friedman, A. Gal, Phys. Rep. 287 (1997) 385.

[3] A. Trzcińska, J. Jastrzȩbski, T. Czosnyka, T. von Egidy, K. Gulda, F.J. Hartmann, J. Iwanicki, B. Ketzer, M. Kisieliński, B. Kłos, W. Kurcewicz, P. Lubiński, P.J. Napiorkowski, L. Pieńkowski, R. Schmidt, E. Widmann, Nucl. Phys. A 692 (2001) 176c.

[4] E. Friedman, A. Gal, Nucl. Inst. Methods in Phys. Research B 214 (2004) 160.

[5] S. Wycech, J. Skalski, R. Smolańczuk, J. Dobaczewski, J. Rook, Phys. Rev. C 54 (1996) 1832.

[6] P. Lubiński, J. Jastrzębski, A. Trzcińska, W. Kurcewicz, F.J. Hartmann, R. Schmidt, T. von Egidy, R. Smolańczuk, S. Wycech, Phys. Rev. C 57 (1998) 2962.

[7] R. Schmidt, F.J. Hartmann, B. Ketzer, T. von Egidy, T. Czosnyka, J. Jastrzȩbski, M. Kisieliński, P. Lubiński, P. Napiorkowski, L. Pieńkowski, A. Trzcińska, B. Kłos, R. Smolańczuk, S. Wycech, W. Pöschl, K. Gulda, W. Kurcewicz, E. Widmann, Phys. Rev. C 60 (1999) 054309.

[8] A. Trzcińska, J. Jastrzȩbski, P. Lubiński, F.J. Hartmann, R. Schmidt, T. von Egidy, B. Kłos, Phys. Rev. Lett. 87 (2001) 082501.

[9] R. Schmidt, A. Trzcińska, T. Czosnyka, T. von Egidy, K. Gulda, F.J. Hartmann, J. Jastrzębski, B. Ketzer, M. Kisieliński, B. Kłos, W. Kurcewicz, P. Lubiński, P. Napiorkowski, L. Pieńkowski, R. Smolańczuk, E. Widmann, S. Wycech, Phys. Rev. C 67 (2003) 044308.

[10] B. Kłos, S. Wycech, A. Trzcińska, J. Jastrzȩbski, T. Czosnyka, M. Kisieliński, P. Lubiński, P. Napiorkowski, L. Pieńkowski, F.J. Hartmann, B. Ketzer, R. Schmidt, T. von Egidy, J. Cugnon, K. Gulda, W. Kurcewicz, E. Widmann, Phys. Rev. C 69 (2004) 044311.

[11] I.N. Mishustin, L.M. Satarov, T.J. Bürvenich, H. Stöcker, W. Greiner, Phys. Rev. C 71 (2005) 035201.

[12] J. Mareš, E. Friedman, A. Gal, Phys. Lett. B 606 (2005) 295.

[13] Y. Sugahara, H. Toki, Nucl. Phys. A 579 (1994) 557.

[14] M.M. Sharma, M.A. Nagarajan, P. Ring, Phys. Lett. B 312 (1993) 377.

[15] E. Friedman, A. Gal, Nucl. Phys. A 658 (1999) 345.

[16] R. Schmidt, F.J. Hartmann, T. von Egidy, T. Czosnyka, J. Iwanicki, J. Jastrzȩbski, M. Kisieliński, P. Lubiński, P. Napiorkowski, L. Pieńkowski, A. Trzcińska, J. Kulpa, R. Smolańczuk, S. Wycech, B. Kłos, K. Gulda, W. Kurcewicz, E. Widmann, Phys. Rev. C 58 (1998) 3195.

[17] G. Fricke, C. Bernhardt, K. Heilig, L.A. Schaller, L. Schellenberg, E.B. Shera, C.W. De Jager, At. Data Nucl. Data Tables 60 (1995) 177.

[18] C.J. Batty, E. Friedman, H.J. Gils, H. Rebel, Advances in Nuclear Physics 19 (1989) 1.

[19] C. García-Recio, J. Nieves, E. Oset, Nucl. Phys. A 547 (1992) 473.

[20] V.E. Starodubsky, N.M. Hintz, Phys. Rev. C 49 (1994) 2118.

[21] A. Krasznahorkay, M. Fujiwara, P. van Aarle, H. Akimune, I. Daito, H. Fujimura, Y. Fujita, M.N. Harakeh, T. Inomata, J. Jänecke, S. Nakayama, A. Tamii, M. Tanaka, H. Toyokawa, W. Uijen, M. Yosoi, Phys. Rev. Lett. 82 (1999) 3216.

[22] B.C. Clark, L.J. Kerr, S. Hamma, Phys. Rev. C 67 (2003) 054605. 
[23] J. Jastrzębski, A. Trzcińska, P. Lubiński, B. Kłos, F.J. Hartmann, T. von Egidy, S. Wycech, Int. J. Mod. Phys. E 13 (2004) 343.

[24] E. Friedman, A. Gal, Nucl. Phys. A 724 (2003) 143.

[25] G.A. Lalazissis, S. Raman, P. Ring, At. Data Nucl. Data Tables 71 (1999) 1.

[26] T. Nikšić, D. Vretenar, P. Finelli, P. Ring, Phys. Rev. C 66 (2002) 024306.

[27] G.A. Lalazissis, T. Nikšić, D. Vretenar, P. Ring, Phys. Rev. C 71 (2005) 024312.

[28] S. Wycech, Nucl. Phys. A 692 (2001) 29c. 


\section{TABLES}

TABLE I. Binding energies $B_{\bar{p}}$ and widths $\Gamma_{\bar{p}}$ (in keV) of deeply-bound $\bar{p}$ atomic states in ${ }^{40} \mathrm{Ca}$ calculated within the static and dynamical approaches using the TM1 RMF parameterization.

\begin{tabular}{lcccc}
\hline \hline$n l$ & static $B_{\bar{p}}$ & dynamical $B_{\bar{p}}$ & static $\Gamma_{\bar{p}}$ & dynamical $\Gamma_{\bar{p}}$ \\
\hline $1 s$ & 1835.132 & 1835.129 & 584.970 & 584.967 \\
$2 p$ & 1516.105 & 1516.101 & 353.299 & 353.300 \\
\hline \hline
\end{tabular}




\section{FIGURES}

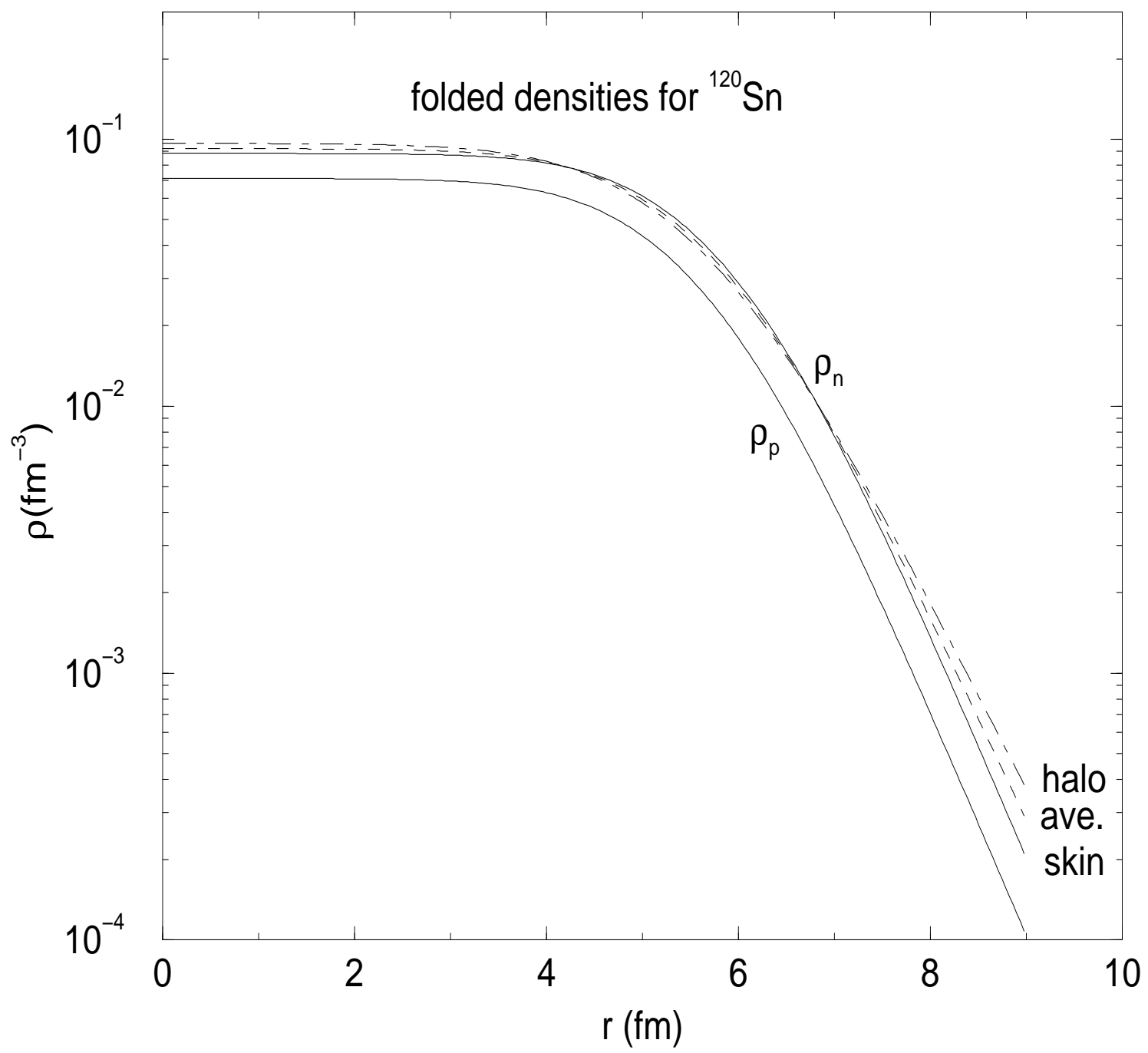

FIG. 1. Proton and neutron densities for ${ }^{120} \mathrm{Sn}$ after a finite range folding with $\beta=0.85 \mathrm{fm}$, see Eq.(9). Neutron densities are calculated with $\alpha=1.2 \mathrm{fm}$, see Eq.(7). 


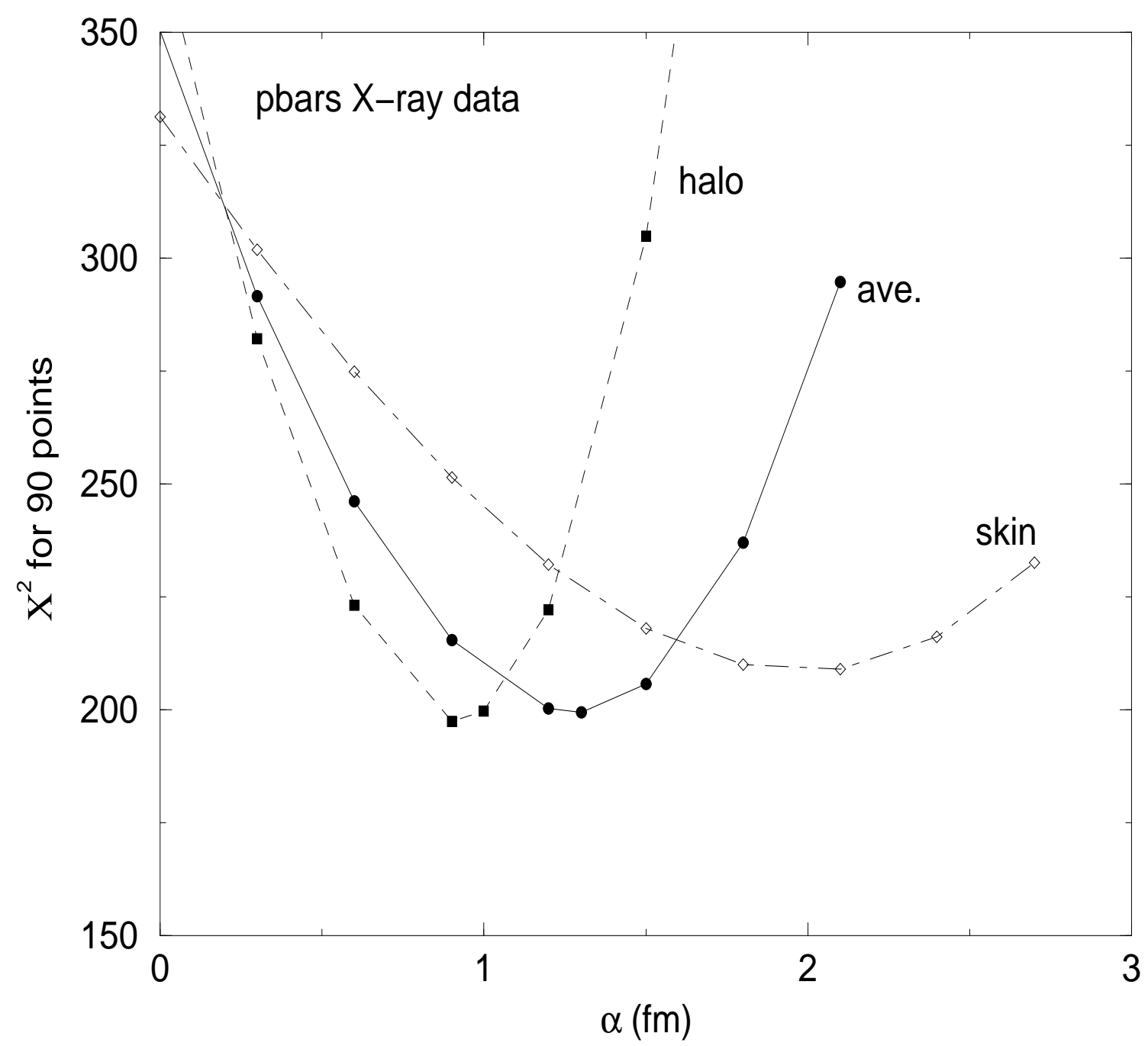

FIG. 2. $\chi^{2}$ values from fits to $\bar{p} \mathrm{X}$-ray data as function of the slope parameter of $r_{n}-r_{p}$ (Eq.(7)) for various types of neutron density. 


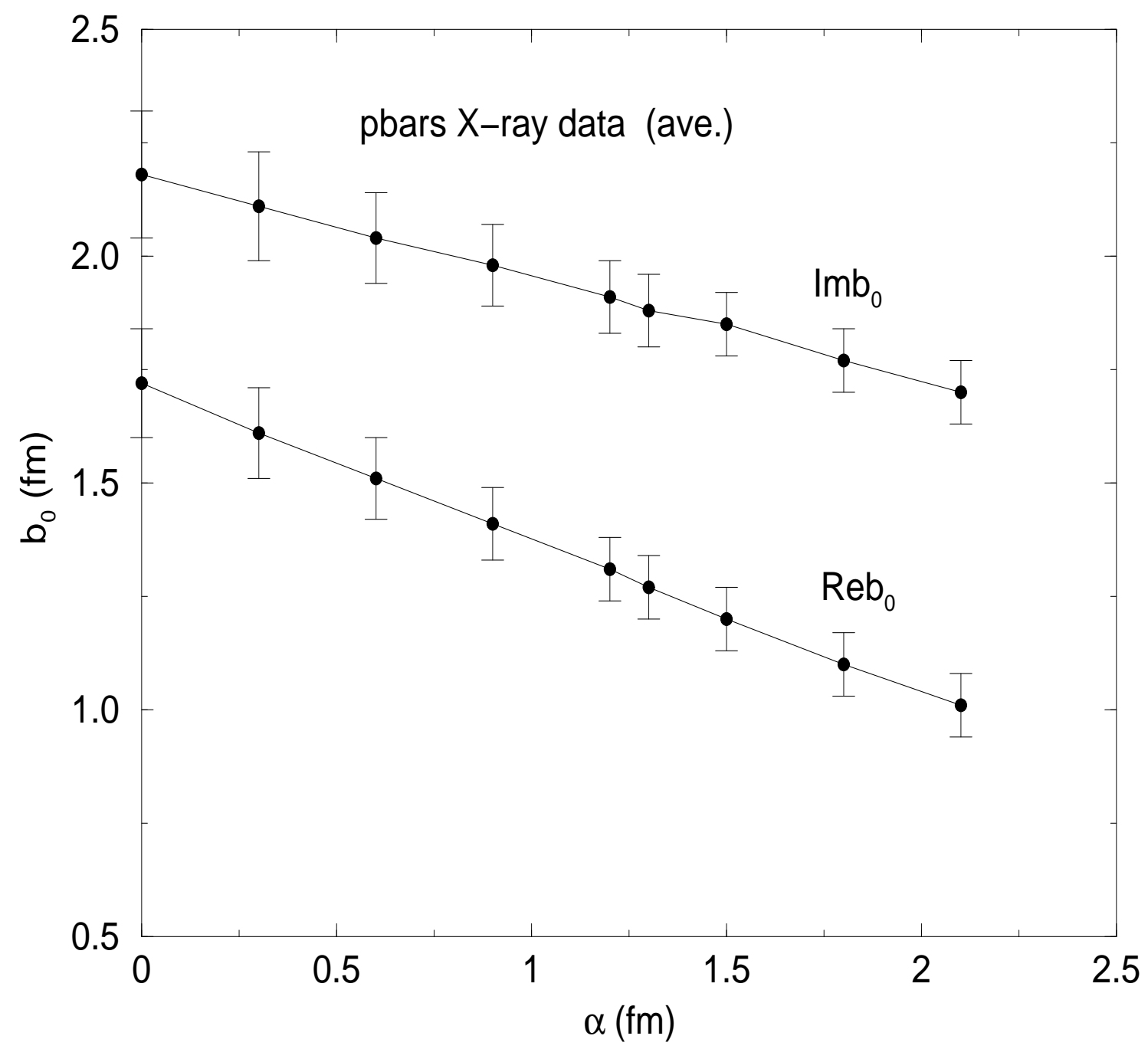

FIG. 3. Values of the potential parameter $b_{0}$ for the 'average' type of neutron density. 


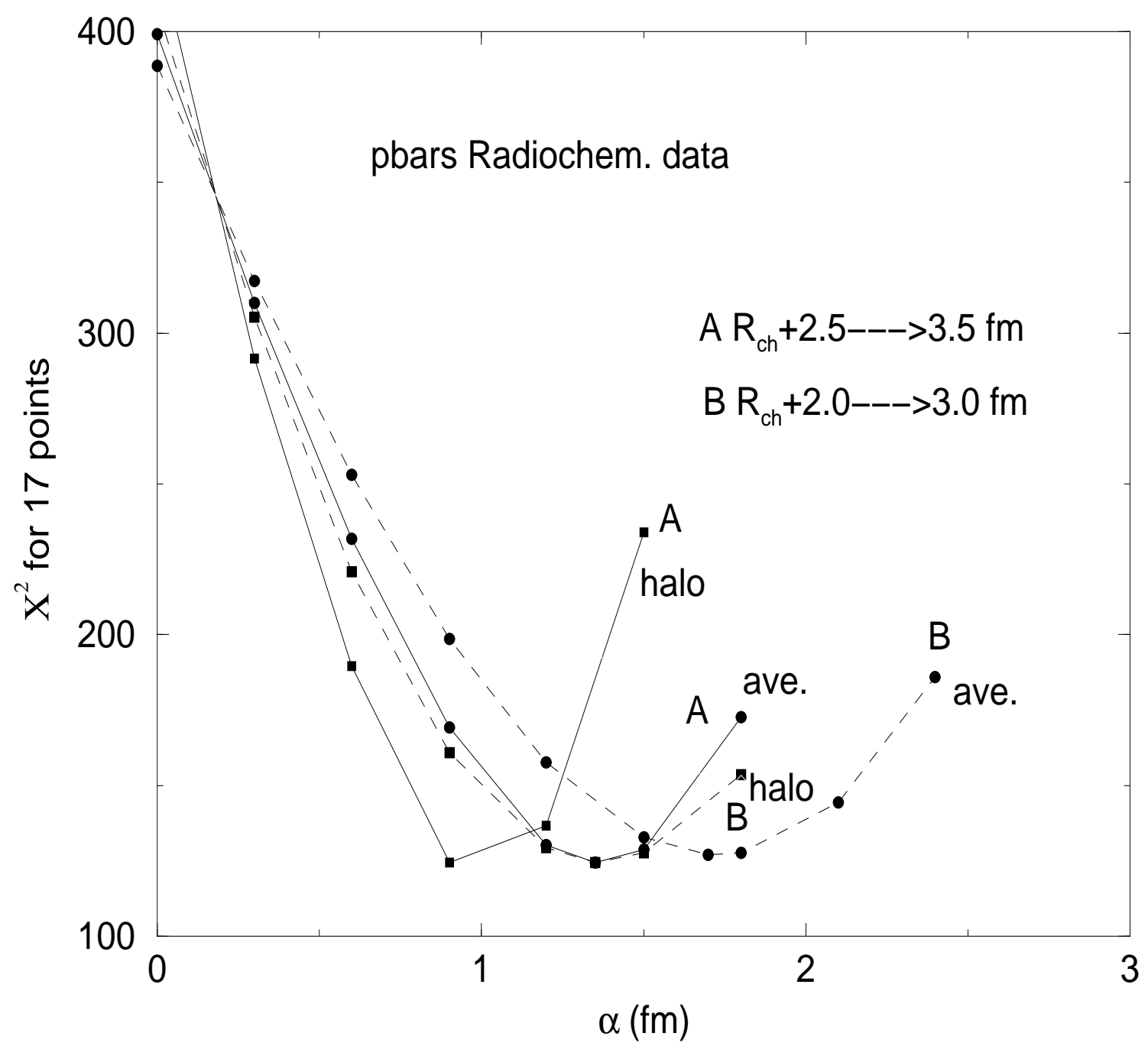

FIG. 4. $\chi^{2}$ values from fits to radiochemical data as function of the slope parameter of $r_{n}-r_{p}$ (Eq.(7)) for 'halo' and 'average' types of neutron density and for two integration ranges: A for 2.5 to $3.5 \mathrm{fm}$ beyond the charge radius; $\mathrm{B}$ for 2.0 to 3.0 beyond the charge radius, see text. 


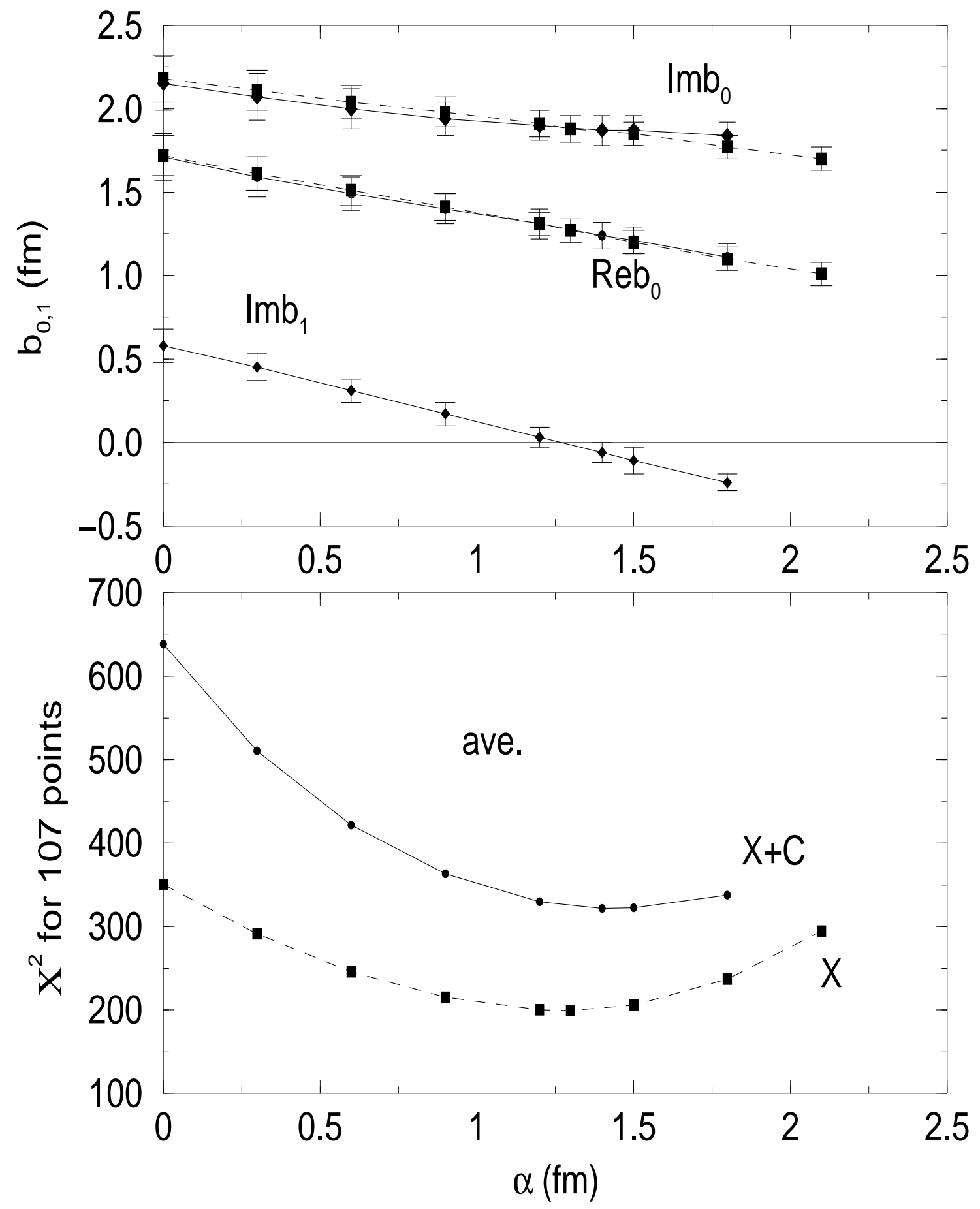

FIG. 5. Results of global fits for the 'average' model of neutron densities. Solid lines for combined X-ray and radiochemical data; dashed lines for X-ray data only. 


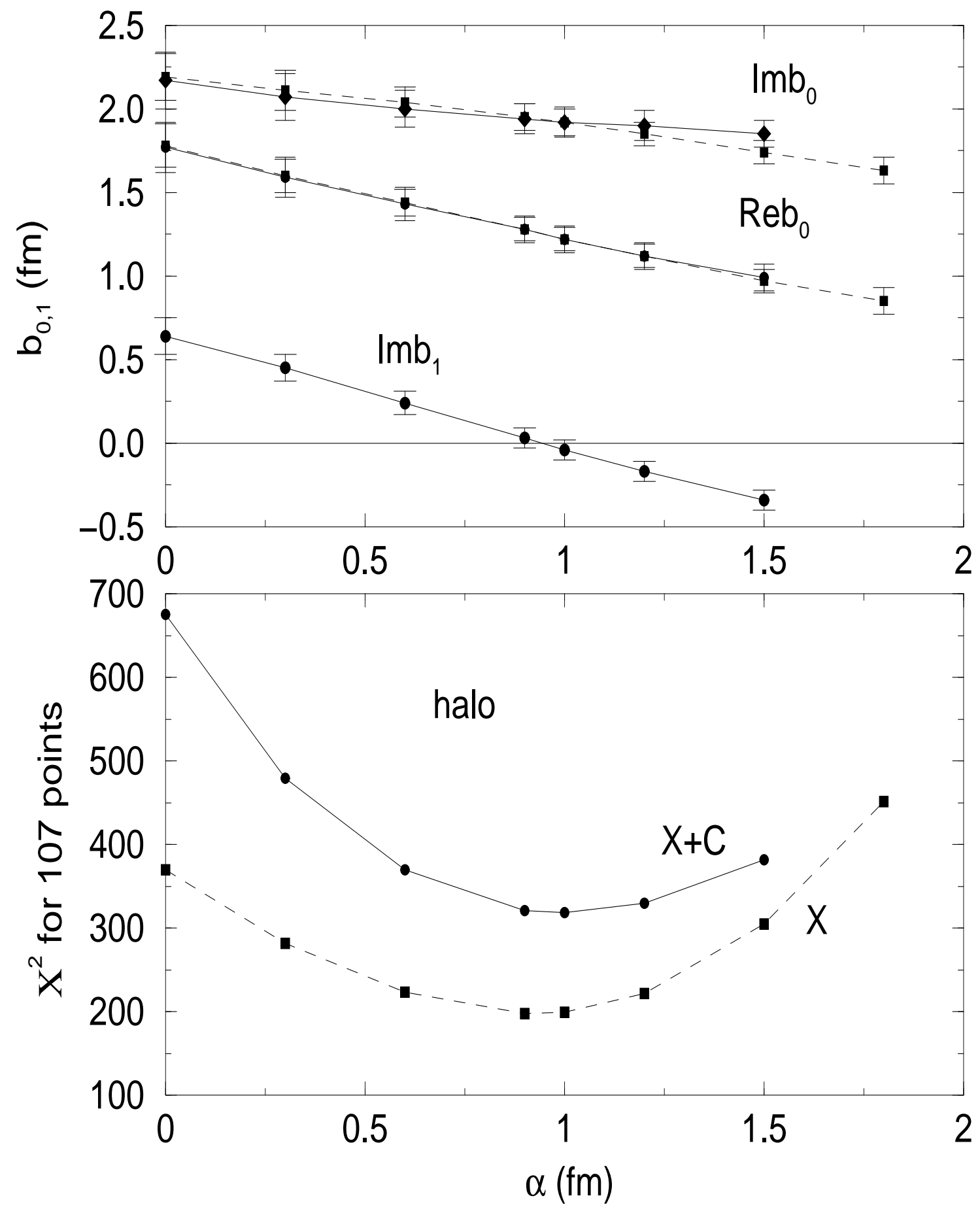

FIG. 6. Same as Fig. 5 but for the 'halo' type of neutron densities. 